\title{
Development of typical elements of silencers for internal combustion transport engines
}

\author{
Anna K unina and Vladimir Tupov* \\ B M STU, Power Engineering Department, 105005, M oscow, Russia
}

\begin{abstract}
A nalysis of various methods for calculating acoustic efficiency of the transport engine noise silencers was carried out, their advantages and disadvantages were considered. Besides, it was shown that acoustic calculation methods, in particular the four-terminal method, could be used at the relatively small noise silencer structure elements cross-section and provide the acceptable accuracy. A nalytical dependence for calculating the noise silencer acoustic efficiency using coefficients of its transfer matrix are provided. Conducted analysis of the transport engine noise silencer design made it possible to develop a number of typical noise-suppressing elements, using which allows to synthesize most of the reactive noise silencers for this purpose. On the example of a specific exhaust noise silencer, a method is shown for its representation in the form of a set of cascade-connected developed typical noise suppressing elements, each of them is described by a corresponding transfer matrix. M ultiplying these matrices, the given noise silencer transfer matrix coefficients could be obtained. The resulting mathematical model of a noise silencer makes it possible to calculate its acoustic efficiency depending on its frequency and opens up a possibility to further optimize the noise silencer design using a computer. Standard elements versatility indicates possibility of their widespread use in calculating and designing various types of vehicle reactive noise silencers.
\end{abstract}

\section{Introduction}

More and more attention is being paid in the last decade to the problem of silencer acoustic calculation. This problem urgency is determined by the fact that majority of domestic vehicles, especially the heavy-load ones, do not satisfy the current standards for internal and external noise. In addition, the current trend towards increasing the vehicle power and speed accompanied by tightening permissible noise levels make the problem of designing highly efficient silencers for the ICE intake and exhaust noise more and more urgent.

Silencer design is often carried out on the basis of experimental studies without any deep research and development study. This approach does not always lead to a desired result; as a result, these silencers require further modification.

In this regard, the task of developing methods for calculating silencers with the given acoustic efficiency meeting requirements for hydraulic resistance, overall dimensions, etc. remain of acute urgency.

* Corresponding author: vvtupov@mail.ru 


\section{Research object, goals and tasks}

According to the principle of operation, silencers are divided into two types: reactive (reflective) and absorption (dissipative). Reactive type silencers are most commonly used, since noise reduction effect there is achieved mainly due to reflection of sound waves passing through them. Along with this, dissipative silencers are also employed, these devices transform acoustic energy into thermal energy and are provided, as a rule, with low hydraulic resistance. In addition, combined silencers are also used, both principles of noise reduction are introduced in their design.

Evaluating advantages and disadvantages in using dissipative and reactive silencers to reduce the transport engines intake and exhaust noise, advantages of the latter should be noted. Dissipative silencers use in the exhaust systems is being impeded due to blowing-out of the sound-absorbing material used by a high-speed gas flow, as well as to contamination of its surface with combustion products, which leads to significant decrease in the noisesuppressing effect. For these reasons, the focus of this work is centered on reactive silencers.

The goal of this work is to develop a computational method for the reactive silencer design, which includes analysis of the device design and creation on this basis of a number of typical acoustically efficient noise-suppressing elements intended for the synthesizing silencers with given acoustic efficiency, as well as taking into account the requirements for acceptable pressure loss and geometrical dimensions.

\section{Analysis of methods for calculating noise silencers}

Certain success was achieved recently in design and development of methods for the silencer acoustic calculation [1-15].

Acoustic methods are widely used in calculations. However, they often give a certain error due to neglecting the averaged gas flow, wide amplitude of sound waves and temperature alterations along the silencer length. It was shown in [5] that acoustic theory could ensure sufficient accuracy in calculations provided that convective gas flow in the silencer elements is taken into consideration.

If transverse dimensions of the silencer elements are small compared to the wavelength, then these elements could be considered as one-dimensional [11]. At the same time, in some elements of the silencer, such as the expansion chamber, resonances may occur determined by the transverse vibration modes. In this case, multidimensional analysis is introduced [12] using the modal method or the finite element method.

Modal method, as applied to the Evaluating advantages and disadvantages in using dissipative and reactive silencers to reduce the transport engines intake and exhaust noise, advantages of the latter should be noted. Dissipative silencers use in the exhaust systems is being impeded due to blowing-out of the sound-absorbing material used by a high-speed gas flow, as well as to contamination of its surface with combustion products, which leads to significant decrease in the noise-suppressing effect. For these reasons, the focus of this work is centered on reactive silencers.

The goal of this work is to develop a computational method for the reactive silencer design, which includes analysis of the device design and creation on this basis of a number of typical acoustically efficient noise-suppressing elements intended for the synthesizing silencers with given acoustic efficiency, as well as taking into account the requirements for acceptable pressure loss and geometrical dimensions.

Modal method, as applied to the engines silencers, is most fully developed in [13]. As a result of calculation, amplitude of each oscillation mode could be determined. Solution is being found numerically and takes time. This method could be applied most successfully to the silencer elements, which geometric shape is described by analytical functions. 
Finite element method could be used to simulate the arbitrary shape silencers [14]. The number of elements, into which the silencer is divided, depends on the required calculation accuracy. This method is labor consuming and requires large computational costs, especially, when taking into account the averaged gas flow.

Analysis conducted showed that each of these methods had their own advantages and disadvantages; therefore, calculation of silencers should be carried out by combined methods based on studying physical processes in them.

\section{Development of reactive silencer typical elements}

Silencer design and optimizing its structure is based on the method of the noise-suppressing system mathematical simulation. In this case, the method of the equivalent four-pole method is widely used, where the sound pressure and the volumetric oscillatory speed at the silencer input and output are interconnected by a transmission matrix with the $A, B, C, D$ coefficients (Fig. 1a,b). These coefficients could be used to calculate the acoustic efficiency of a designed silencer:

$$
I L=20 \lg \left[\sqrt{\frac{\operatorname{Re}\left(Z_{r 1}\right)}{\operatorname{Re}\left(Z_{r 2}\right)}} \cdot\left|\frac{A Z_{r 2}+B+Z_{s}\left(C Z_{r 2}+D\right)}{Z_{r 1}+Z_{s}}\right|\right], \mathrm{dB},
$$

where: $Z r_{1}$ is the sound energy radiation impedance by the end opening of the ICE exhaust system without a silencer; $Z_{r}$ is the sound energy radiation impedance by the silencer end opening; $Z_{s}$ is the noise source acoustic impedance; $A, B, C, D$ are the complex coefficients of the silencer transmission matrix.

a)

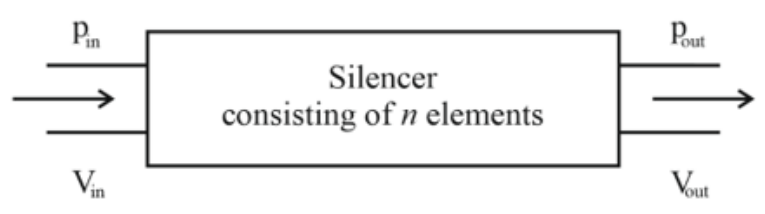

b)

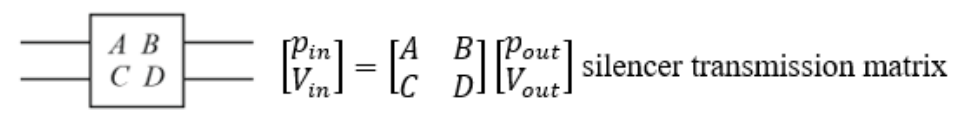

c)

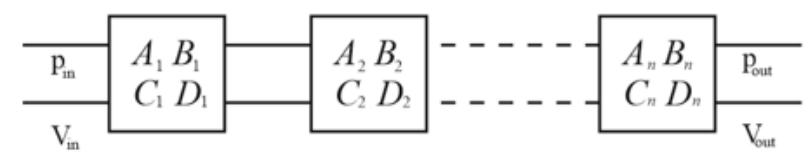

$\left[\begin{array}{ll}A & B \\ C & D\end{array}\right]=\left[\begin{array}{ll}A_{1} & B_{1} \\ C_{1} & D_{1}\end{array}\right]\left[\begin{array}{ll}A_{2} & B_{2} \\ C_{2} & D_{2}\end{array}\right] \cdots\left[\begin{array}{ll}A_{n} & B_{n} \\ C_{n} & D_{n}\end{array}\right] n$ silencer elements

Fig. 1. Caption of the Figure 1. Below the figure.

Rational synthesis of silencers should be provided from correctly selected and (or) newly created noise-suppressing elements during design in order to ensure the silencer required acoustic efficiency in a given frequency range. Silencer elements can be represented by equivalent quadripole whose transmission matrices are known or subject to calculation. Fig. 1C shows an equivalent silencer circuit consisting of $\mathrm{N}$ cascaded quadripole, each of which simulates a particular structural element. The transmission matrix of the silencer in this case is obtained by multiplying the transmission matrices of its component components.

At first glance, development of transmission matrices for the vehicle silencer elements knowing their wide variety could appear an almost insoluble task. However, conducted 
analysis of domestic and foreign silencers made it possible to select a limited number of typical elements, using which most of the highly efficient silencer designs could be synthesized. Vehicle silencer elements are divided into two classes: containing nonperforated and containing perforated noise-suppressing elements. This paper considers nonperforated typical elements and perforated partition.

Most of the obtained typical elements of reactive silencers are schematically shown in Table 1. Each element is marked with a number, which is further used in the silencer design diagram.

Table 1. Reactive silencer typical elements diagram.

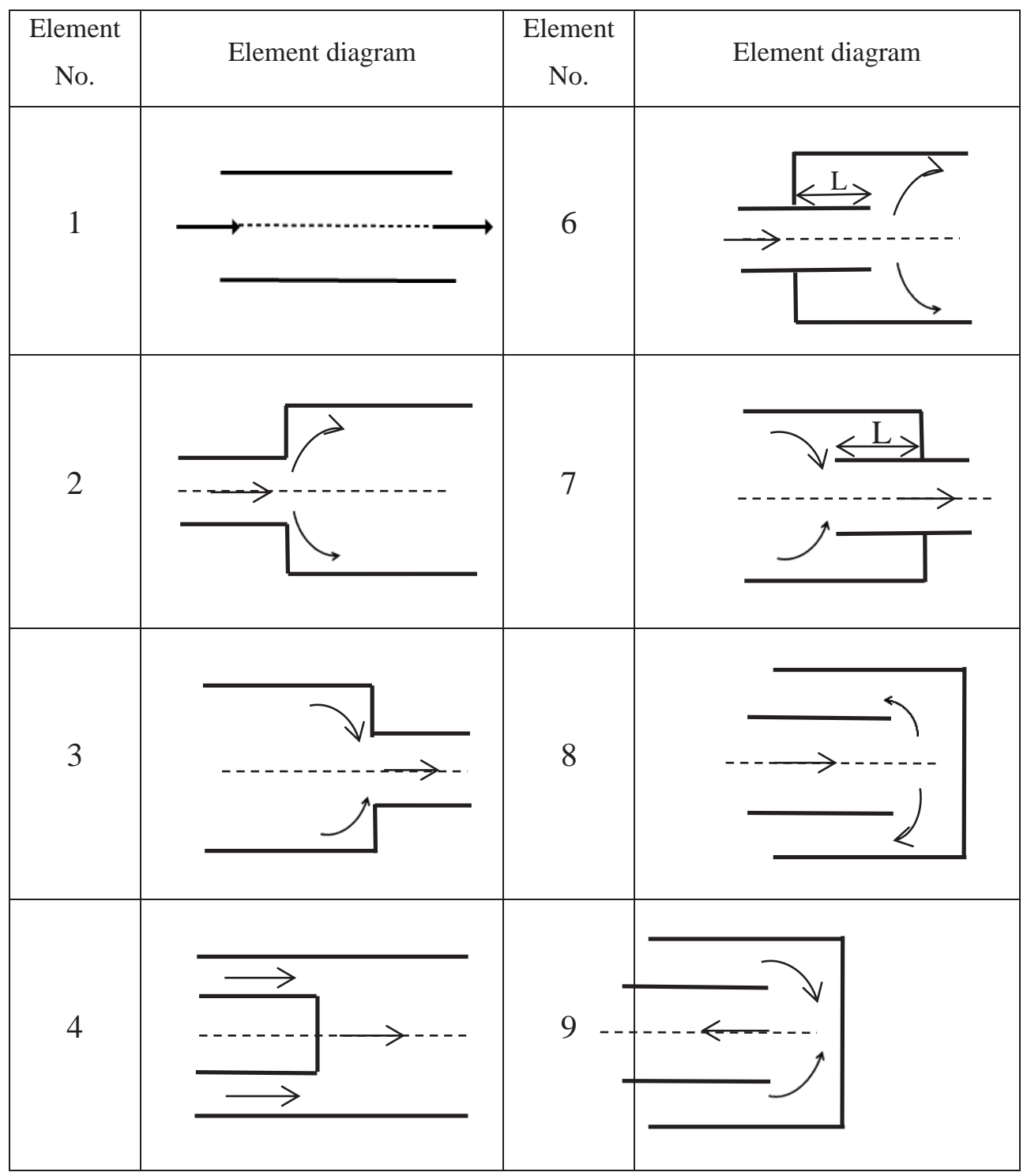




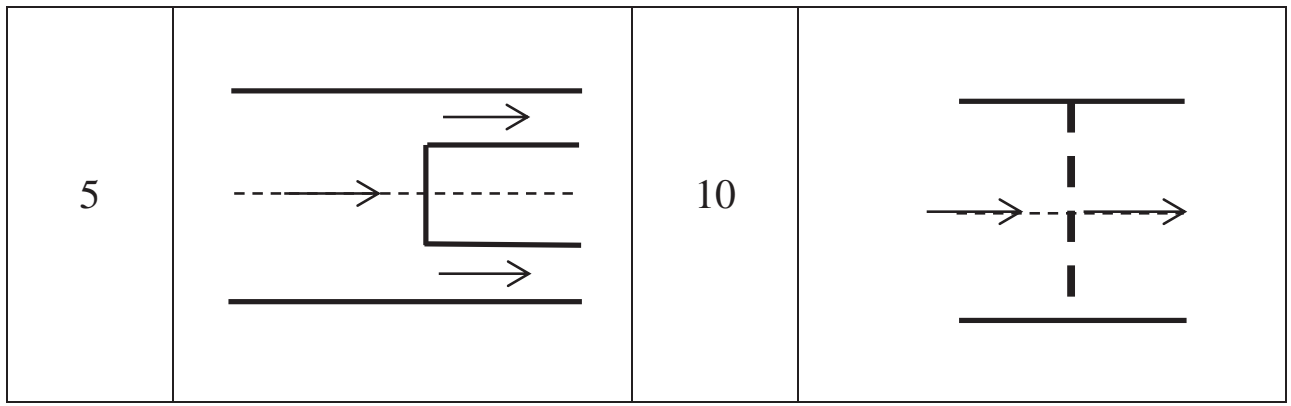

Constant section waveguide opened at both ends is the most common element in silencers. Under the plane wave motion condition, transmission matrix of a waveguide with an arbitrary flow section shape and accuracy to the loss of acoustic energy is similar to the transmission matrix of a straight pipe with circular cross section (element 1 in the table).

Abrupt alteration in the waveguide section (expansion - typical element 2 or contraction - element 3) leads to reflecting part of the sound wave back to the source and, thereby, to a decrease in the transmitted wave amplitude compared to the amplitude of the incident one. When creating the transmission matrix, added mass and irreversible energy losses in the zone of the cross-section variations should be taken into consideration.

Abrupt waveguide section alteration could be constructively implemented by inserting a plug with flat closed end (elements 4 and 5 in the Table). Due to the changing nature of flow in the cross-section jump zone, new values of the added mass and losses could be expected that are differing from elements 2 and 3.

When the pipe is inserted into the expansion chamber (elements 6 and 7 in the Table), conditions of the flow significant expansion and contraction differ from those already considered. Compiling the transfer matrix for these elements would require to take into account the chamber section with the L length closed at one end and connected parallel to the sound flow passing through the waveguide. Elements 8 and 9 are similar in the operation principle, where the flow rotates by 180 degrees, and it is reflected in alteration of the added mass and active losses in the area of the narrow waveguide opening.

Perforated partitions (item 10 in the Table) are also used in silencers. A special case of such an element could be a flat partition with a single opening.

\section{Application Typical elements in developing the silencer calculation diagram}

Creation of the silencer design diagram should be started with the analysis of its structure. In this case, it is necessary to divide the silencer under study into separate components so, that each of them could be approximated by a typical element. The essence of this method could be illustrated by the example of a silencer analysis schematically shown in section in Fig. 2. 


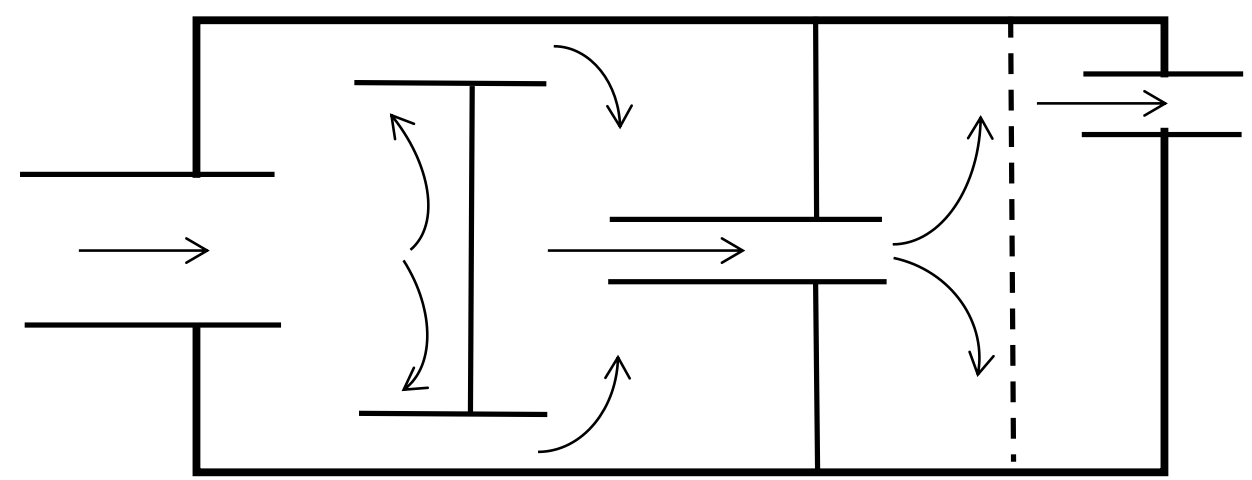

Fig. 2. Transport engines exhaust silencer structural diagram e.

Sound waves entering the silencer through the input opening are propagating by the input pipe (typical item 1 in the Table). Then, the waves exit from the pipe end opening with a turn (typical element 6), their front expands, and they propagate inside the chamber cylindrical section (typical element 1 ) to the entrance to the annular channel entrance (typical element 7). Then they move along it (typical element 1 ) to the output opening, and from there they enter the second chamber expanding (typical element 6). Further, the waves propagate along the cylindrical section of the second chamber (typical element 1), and then come to the input (typical element 7) of the next pipe and propagate along it (typical element 1). From the pipe end opening, the waves pass into the third expansion chamber (typical element 6), move along its cylindrical section (typical element 1) to the perforated partition. Passing through perforation, the waves are exposed to contraction and then to front expansion (typical element 10). Afterwards, they move along the cylindrical section of the third chamber (typical element 1) to the end pipe entrance. The wave front narrows here, and they enter the input (typical element 7) of the silencer end pipe, propagate along it (typical element 1) and exit into the surrounding environment.

Conducted silencer analysis (Fig. 2) showed that its design diagram could be represented by a set of standard elements connected in series (Fig. 3) and presented in Section 3.

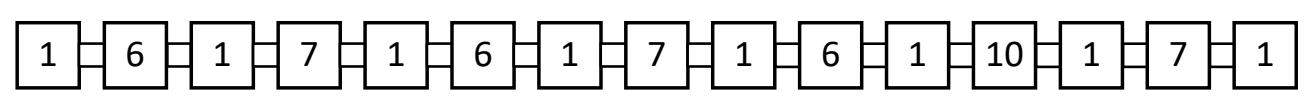

Fig. 3. Analyzed silencer design diagram composed of typical elements.

The resulting design diagram could be used to calculate the transmission matrix of a given silencer by multiplying transmission matrices of its constituent elements. Substituting the silencer transmission matrix coefficients in formula (1), insertion loss (in other words, effect of the silencer installation) could be calculated depending on the sound frequency. The resulting silencer mathematical model makes it possible to calculate its acoustic efficiency depending on the frequency and opens an opportunity to further optimize the silencer design using a computer.

Introduction of standard elements makes it possible to significantly simplify the silencer design scheme and reduce the amount of calculations in calculating its acoustic efficiency. Standard elements versatility indicates possibility of their widespread use in calculation and design of the vehicle reactive silencer various structures. 


\section{Conclusion}

A number of typical-noise suppressing elements were developed, using which it is possible to synthesize most of the vehicle reactive silencers. Introduction of standard elements makes it possible to significantly simplify the silencer design diagram and reduce the amount of calculations in determining its acoustic efficiency. The developed silencer standard elements are the basis for further work, including development of their mathematical models and completion of the computational method for designing the vehicle silencers.

\section{References}

1. M . L. M unjal, Acoustics of ducts and Mufflers, New Y ork: W iley-Interscience, 1987. 328 (1987)

2. R. N. Starobinsky, Silencers, Technical acoustics of transport machines: Handbook, SPb.: Polytechnic, 1992, 200-265 (1992)

3. V. V. Tupov, E. G. B angoyan, Investigation of acoustic characteristics of silencers of exhaust noise from the motor transport internal combustion engines, Safety in technosphere. INFRA -M Scientific Publishing Center LLC (M oscow), issue 43, 2013, 30-35 (2013)

4. Y u. L. Tkachenko, Development and implementation of acoustic calculation methods for transport vehicle reactive silencers, A bstract, M oscow, 1998, 23 (1998)

5. R. J. Alfredson, P. O. A. L. Davies, Performance of Exhaust Silencer Components, Journal of Sound and V ibration. 1971, 15(2), 175-196 (1971)

6. S. V. B elotserkovsky, V. E. Tolsky, Automobile silencers: modern requirements, development trends, methods of calculation and testing, Technical A coustics el ectronic journal. http://ejta.org/ru, 1 (2001) 4.1-4.8. (2001)

7. A . I. Komkin, A. A . A grafonova, S. I. Yudin, Assessment of the acoustic efficiency of automobile noise silencers, Safety in the technosphere, 2012, N 0. 3, 61-65 (2012)

8. V. A. Kulkina, V. V. Tupov, Assessment of the acoustic efficiency of the automobile noise reactive silencers. Overview in proceedings of the Third A II-R ussian A 44 Conference of $Y$ oung Scientists and Specialists "A coustics of the Environment" (A SO2018), 144-147 (2018)

9. Y u. L. Tkachenko, V. V. Tupov, Method of element-by-element mathematical simulation of reactive noise suppressors, Ecological systems and devices, N 0. 1,11-14 (2000)

10. A . I. Komkin, V. V. Tupov, On calculation of acoustic characteristics of noise silencers. BM STU Bulletin. Series M echanical engineering, N o. 3, 118-124 (1994)

11. J. W. Sullivan, Simulation of engine exhaust system noise, A erodynamic noise in technology: Tr. from English. / Ed. S.L. V ishnevsky. - M .: M ir, 1983, 233-256 (1983)

12. L. J. Eriksson, Effect on Inlet/Outlet Location on Higher Order Modes in Silencers, J ournal of the A coustical Society of A merica, 72(2), 1208-1211. (1982)

13. R. N. Starobinsky, Theory and systems of noise silencers for gas exhaust systems of internal combustion engines, dis. doc. tech. sciences: 05.04.02 / TPI. - Togliatti, 1982, 333 (1982)

14. G. I. J. Y oung, M. J. Crocker, Prediction of Transmission Loss in Mufflers by the Finite Element Method, Journal of the A coustical Society of A merica, 57(1), 144-148 (1975) 
15. V.M. Kondrashov, Yu.S. Grigoriev, V.V. Tupov et al., Two-stroke carburetor internal combustion engines, M oscow: M ashinostroyenie, 272 (1990) 